\title{
Correspondence
}

\section{Blame the 'blame culture'}

Mukherjee et al identify several important factors which discourage young doctors from choosing to train as psychiatrists. Stigma, low-quality undergraduate training and a perception that the specialty is unscientific probably all play a part. However, I contend that these factors are likely to have been influencing these doctors for many years. Why a recruitment crisis now?

I disagree that the recent reduction in the number of training posts in academic psychiatry is an important influence on most doctors' career choice. However, I agree that the lack of psychiatry F2 posts and the introduction of Modernising Medical Careers have probably been influential.

Sadly, the most important influence is the paucity of consultant psychiatrists who act as good role models. Medical students and junior doctors notice the absence of enthusiastic senior colleagues. I agree that New Ways of Working has undermined consultant morale, probably to a significant extent, but the most important issue is the stress caused by the culture of repeated inquiries, which follow untoward incidents. Most other medical specialties are not subject to the same intensive 'blame culture'. The recruitment crisis will continue until this issue is addressed.

1 Mukherjee K, Maier M, Wessely S. UK crisis in recruitment into psychiatric training. Psychiatrist 2013; 37: 210-4.

Keith E. Dudleston, consultant psychiatrist (retired), Ivybridge, UK, email: tp@rcpsych.co.uk

doi: $10.1192 / p b .37 .8 .276$

\section{Why psychiatry isn't sexy ...}

I am 2 weeks into my psychiatry placement, and have been forced to ask myself some uncomfortable questions. ${ }^{1}$ As many of my fellow students, I began with a negative view of psychiatry based almost entirely on our formal (and more importantly, informal) medical training. Although I am increasingly aware of the many compelling arguments which challenge our prejudices, unfortunately, the rot sets in before we have even stepped into a ward.

It is hard to establish exactly when this happens. Mental illness is stigmatised by friends. It makes us feel uncomfortable. We don't understand it. The mechanisms underlying many mental illnesses seem so vague as to fall into the realms of pseudoscience. Medical schools select scientists. We want to know how, and why, and what will cure. Psychiatry dances at the edge of such parameters. We are taught by academics that the drugs don't work; well, they do, but they have intolerable side-effects. Electroconvulsive therapy works, but we don't know how, and anyway, seizures are generally a bad thing.

Archdall et $a l^{2}$ emphasised the importance placed on role models. It is a well-known 'fact' among medical students that psychiatrists are 'crazy'. This is unfair. Lots of general practitioners are crazy too. Surgeons must be crazy to endure the training they actively choose. This view may prevail because while roaming around the hospital we come into contact with many more generic surgeons and medics than we do psychiatrists. Psychiatric wards and units are 'other'. We rarely (never) see psychiatrists at the medical grand round.

For the inexperienced, psychiatric patients are scary! It is humiliating enough when the cantankerous gentleman on the respiratory ward tells you to, ahem, go away. Infinitely worse when there is the slim possibility that the psychotic patient 'could kill you, ha-ha ... !'. Our communication skills session came halfway through the psychiatric placement. This lack of preparation seems to be a common feature of UK medical education, and it compounds the unease.

Most medical students see themselves graduating to wander around wards swinging stethoscopes and healing the sick; delivering babies; curing cancer in theatre; entering academia and naming a transcription factor after themselves; or at worst, serving a broad range of ailments in the community and thereby having a life. We need more exposure to this diverse branch of medicine to challenge our idea of what 'doctor' means.

The psychiatry ward round was a revelation to me. High-profile, successful psychiatrists would provide us with role models that challenge our misconceptions. In the past 2 weeks I have encountered some dedicated and inspiring clinicians, I have met some fascinating patients with interesting and complex conditions, and I have had my eyes opened to the overlooked yet vitally important value that psychiatry brings to the clinical care of a whole range of people. Popular opinion might dictate that psychiatry isn't sexy, but it's on my list.

1 Mukherjee K, Maier M, Wessely S. UK crisis in recruitment into psychiatric training. Psychiatrist 2013; 37: 210-4.

2 Archdall C, Atapattu T, Anderson E. Qualitative study of medical students' experiences of a psychiatric attachment. Psychiatrist 2013; 37: $21-4$.

Nicky Jecks, 5th year medical student, University of Cambridge, UK, email: nj250@cam.ac.uk

doi: $10.1192 / \mathrm{pb} .37 .8 .276 \mathrm{a}$

\section{Medical students are susceptible to the public image of psychiatry}

Mukherjee and colleagues ${ }^{1}$ concentrate mainly on interventions in medical training, but it is also important to bear in mind that, as members of the public, medical students are constantly exposed to public perceptions and media portrayals of psychiatry. The poor public perception of psychiatry is perhaps a more fundamental reason for poor recruitment.

Surveys indicate psychiatrists are perceived as having low status compared with other doctors, with psychiatry being seen as relatively non-scientific, non-medical and ineffective. Relatively few people know the difference between psychiatry, psychology, psychotherapy, psychoanalysis and general counselling, and relatively few know that psychiatrists are doctors. A survey showed that $70 \%$ of people view electroconvulsive therapy as harmful and only $7 \%$ view it as potentially helpful; many have a highly negative view of any psychotropic medication, and have too high 